\title{
Visualization on the Web of 20 Years of Crop Rotation and Wildlife Co-Evolutions
}

\author{
Christine Plumejeaud-Perreau, ${ }^{\mathrm{a}}$ Cyril Poitevin, ${ }^{\mathrm{a}}$ Vincent Bretagnolle, ${ }^{\mathrm{b}}$ \\ ${ }^{a}$ LIENSS, UMR 7266 CNRS, 17000 La Rochelle, France - (cyril.poitevin, christine.plumejeaud-perreau)@univ-lr.fr \\ ${ }^{b}$ CEBC, UMR 7372 CNRS, 79360 Villiers-en-Bois, France-breta@cebc.cnrsfr
}

\begin{abstract}
The accumulation of evidences of the effects of intensive agricultural practices against wildlife fauna and flora, and biodiversity in general, has been largely published in scientific papers (Tildman, 1999). However, data serving as sup-port to their conclusions are often kept hidden behind research institutions. This paper presents a data visualization sys-tem opened on the Web allowing citizens to get a comprehensive access to data issued from such kind of research institution, collected for more than 20 years. The Web Information System has been thought in order to ease the comparison of data issues from various databases describing the same object, the agricultural landscape, at different scales and through different observation devices. An interactive visualization is proposed in order to check co-evolution of fauna and flora together with agricultural practices. It mixes aerial orthoimagery produced since 1950 with vectorial data showing the evolutions of agricultural parcels with those of a few sentinel species such as the Montagu's harrier. This is made through a composition of maps, charts and time lines, and specific tools for comparison. A particular concern is given to the observation effort bias in order to show meaningful statistical aggregates.
\end{abstract}

Keywords: Interactive data visualization, environmental data, spatio-temporal analysis

\section{Introduction}

This research aims at identifying effective solutions for integrating agricultural development and conservation of biodiversity at the landscape scale. Various environmental data have been collected with three main objectives. The first considers intensive monitoring of landscape structures and all biodiversity components. The second objective concerns the experimental investigation of some ecosystem services within the agro-ecological framework, with experimental manipulation of pollination and biological control, confronted to crop production and socio-economic value of farming. The third aim is directly targeted to stakeholders, through dissemination of research, citizen science as well as intervention research. In this latter category, we investigate how to sensitize local policy makers and citizens for a collective management of crop rotation that would maximize ecosystem services. One of our hypotheses is that a free Web ac-cess to a set of interactive visualizations staging the collected data can give a comprehensive and direct overview of facts linked to the agricultural practices.

The major problematic is to give comprehensive pieces of information to a non-expert user using a set of data that are very heterogeneous, and rather not simple to understand. Our information system tries to address this challenge, and we detail in this contribution what kind of environmental data are analyzed and the Web design of this system.

\section{Description of the environmental data}

All data are issued from the ecological monitoring of a study area, of $450 \mathrm{~km}^{2}$, located in the south-west of France, certified as "Zone Atelier". As a research infrastructure managed by CNRS, each "Zone Atelier" targets a scientific contribution to questions of sustainable development, in a perspective of use and long-term sharing of resources, spaces and territories (Leveque et al. 2000). More than half of the study site has been designated as a NATURA 2000 site in 2003. Road mesh and tree hedges, that play a fundamental role in natural habitats structure, have all been digitalized by photointerpretation in 2005 of orthoimagery, and updated with 2011 and 2014 data. Furthermore, a set of aerial photos taken in 1958, 1969, 1982, 1990, 2002, 2007 and 2011 have been georeferenced and assembled. Put side by side, those images give an astonishing view on the evolution of the area with the systematic extension of the area of agricultural parcels and the reduction of their number and their diversity (Fig. 1).

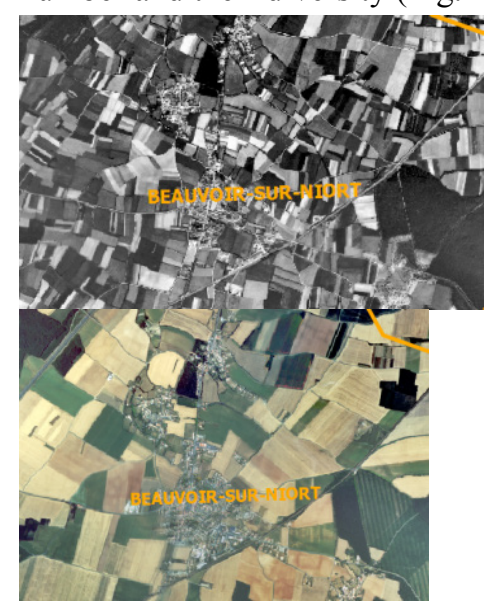

Fig. 1. Changes around Beauvoir between 1958 and 1990.

The monitoring of agricultural parcel has required the agreement of farmers and has been submitted to a 
declaration to "Commission Nationale de l'Informatique et des Libertés" (CNIL), a French commission protecting data privacy, thus data must remain non-public. This must be taken into account in our visualization and dissemination problematic.

In addition to these data, various databases about fauna and flora have been constituted. The observation effort has not been consistent within years, which should not be misinterpreted as a disappearance or increase of the fauna of flo-ra species's abundance. Thus statistical charts and discretization of values in maps have to take into account this fact and should display figures proportionate to the yearly observation effort.

\section{A GIS on the Web for Environmental Data}

This application aims to give an equal importance to aerial photographs and ground collected data, in order to ad-dress both the need for attractive data easier to understand for users like the aerial photographs, and the need for a deeper knowledge of the biodiversity evolution and crop evolution that can be explained by ground data. We propose a conception using a set of many tabs in the Web site, allowing user to navigate between those data, with one page dedicated to the aerial imagery, and one page dedicated to a synthesis of ground collected data.

The page dedicated to aerial photographs aims to ease the comparison of different temporal states of the land-scape. A tool bar at the top of the map allows the access to various representations.

The first representation is an animation with a time bar at the bottom of the map and a play button to launch the animation showing the landscape at successive dates (1958 - 1969 - 1982 - 1990 - 2002- 2007 - 2011).

The second representation is dedicated to comparison tasks. User can select 2 dates for orthophotographs and use one of the two following options. The magnifying option called in French 'Disque de transparence', coming with the OpenLayer3-1 implementation, allows for a view on the past anywhere inside a map, using a kind of magnifying glass. The swiping option called in French 'Juxtaposition dynamique' allows horizontal comparisons by juxtaposing two snapshots of the landscape. Both options are very playful, and act like very intuitive toys, easy to use and configure.

The design of the page dedicated to a synthesis of ground collected data gives equal importance to temporal and spatial dimension. It aims at presenting simultaneously both the evolution through time and the dispersion in space of various species. Thus the screen is split in 2 panels, one for the map on the left, and one for the charts on the right, but with a common temporal dimension driving the data display (Fig. 2). The figure 2 is an example exploring alfalfa distribution (luzerne in French) on the grid zoning, for 2009 year, and its evolution in quantity in time.

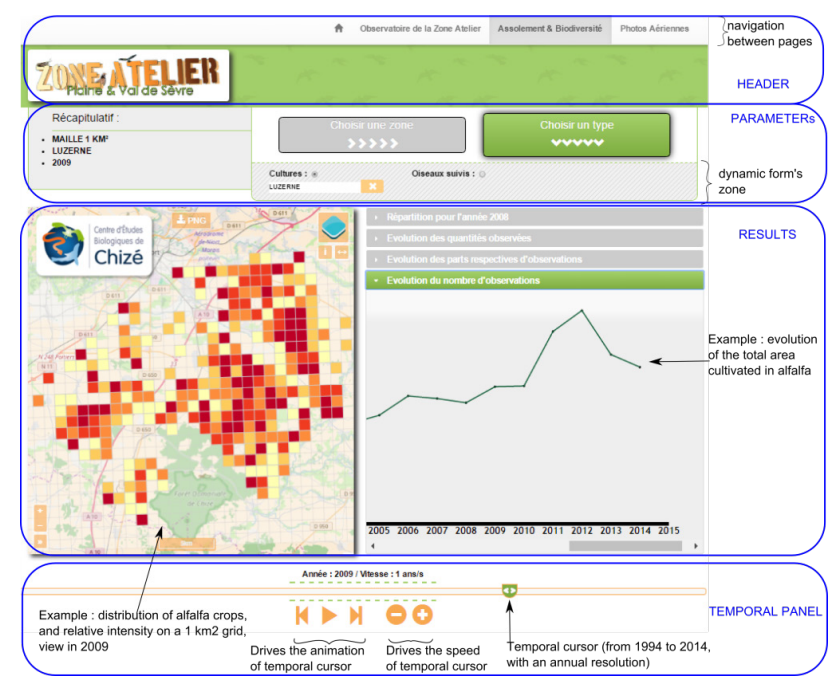

Fig 2. Viewing the alfalfa evolution through the Web interface.

In our mind, the most valuable of this research resides in the way statistics are presented. In fact, there is a big concern about the figures and the statistics (the aggregates) we can explore, in many fashions, in order to have a comprehensive view on the phenomena. As said in the previous section, a bias automatically exists in ecology due to the fact that observed samples may not be representative of the full population distribution (Martinez et al., 1999). Notwith-standing the bias, we would only show evolution of the observation effort. That's why all figures of maps (for crops or species) have always been computed relatively to the observation effort, by giving relative ratios to the total amount of observations and the surveyed area. Furthermore, map discretization is dynamically computed according the selected thematic variable and the zoning scale in order to have the same cuts through time.

\section{Conclusion}

In this proposal, we have described a GIS on the Web designed in the perspective of giving access to citizens to very important environmental issues around the agricultural practices. In the flow of the data visualization movement, we want to give them ways to explore very original datasets, in an easy and simple way. The challenge is that those data are under diffusion restrictions, and that many dimensions have to be explored in the same time to get a comprehensive view on the measured phenomena.

After a look on what is currently available in term of Web sites for those kinds of data in Europe, after a review of all the new technologies that could be of help, we have decided to design our own Web interface. The accent is put on the easiness and the attractiveness of the proposed representations. Our concern is also about the veracity and the confidentially of the data in order to diffuse aggregates meaningful for the user. This means hiding the bias due to the variations of the observation effort through time.

The result implemented in a Web site (http://zageminat.cnrs.fr:82/) that is currently accessed by many 
citizens seems to us a success. The site itself is being referenced by the official Web site of the Zone Atelier whose URL is http://www.za.plainevalsevre.cnrs.fr/. In particular, a page entitled "espace-grand-public" presents our interactive visualization device on the Web, introducing (in French) the meaning of our representations.

\section{References}

Lévêque, C., Pavé, L., Abbadie, L., Weill, A, Vivien, FD., (2000). Les zones ateliers, des dispositifs pour la recherche sur l'environnement et les anthroposystèmes. Natures, Sciences, Sociétés 8, 43-52.

Martinez, N. D., Hawkins, B. A., Dawah, H. A., Feifarek, B. P. (1999) Effects Of Sampling Effort on Characterization of Food-Web Structure, Ecology, DOI: 10.1890/0012-9658(1999)080[1044:EOSEOC]2.0.CO;2

Tilman, D. (1999), The ecological consequences of changes in biodiversity: A search for general principles. Ecology, 80: 1455-1474. doi:10.1890/00129658(1999)080[1455:TECOCI]2.0.CO;2 